\title{
Stock Indices for Emerging Markets
}

\author{
K. Karpio ${ }^{a}$, A.J. Orlowski ${ }^{a, b}$ And P. EUKASIEWICZ ${ }^{a}$ \\ ${ }^{a}$ Katedra Informatyki SGGW, Nowoursynowska 166, 02-787 Warszawa, Poland \\ ${ }^{b}$ Instytut Fizyki PAN, al. Lotników 32/46, 02-668 Warszawa, Poland
}

\begin{abstract}
Indices of selected financial markets from various parts of world, different sizes and levels of development are investigated. The local Hurst exponent is globally compared to log-prices. Periodic changes in correlation coefficient are quantified via discrete Fourier transform. Local Hurst exponents spectra are discussed for investigated markets.
\end{abstract}

PACS numbers: 89.65.Gh, 02.30.Nw

\section{Introduction}

In the last years a lot of attention was paid to the Hurst exponent and its application to predict prices in financial markets. In this study, we employ the Hurst exponent to quantify the long-term correlations. We use detrended fluctuation analysis (DFA) method to calculate the Hurst exponent, as it is known to be the most efficient [1]. The method itself is described in the next section. The application of DFA for studying the stock market behavior was initiated by Liu et al. [2]. They show that the S\&P500 stock index exhibits weak long-range correlations. On the other hand, Grech and Pamuła [3] and Grech and Mazur [4] employed the local Hurst exponent behavior in order to predict crashes on the financial market.

\section{Methodology}

The methodology employed to process a data is based on applications of the DFA to the return time series. This is the fractal scaling method commonly applied for detecting long-range correlations in non-stationary sequences. The method of calculating the Hurst exponent can be summarized as follows. For a given stochastic time series $p(i), i=1, \ldots, M$, which in our case is a series of index daily return rates: $p(i)=\ln \left(x_{i+1}\right)-\ln \left(x_{i}\right)$, $i=1, \ldots, M-1$, where $x_{i}$ is an $i$-th closure price, compute the mean return rate $\bar{p}=\left[\sum_{i=1}^{\mathrm{M}-1} p(i)\right] /(M-1)$. Then calculate the integrated time series given by the equation below

$$
x(i)=\sum_{j=1}^{i}[p(j)-\bar{p}], \quad i=1, \ldots, M-1 .
$$

Now, let us work with the observation box of $N=$ 210 consecutive return rates $(N$ is called the width or observation box) that implies the idea of a local Hurst exponent. The size of the observation box $N$ should be set carefully. If $N$ is too large, $H_{\text {loc }}$ losses its locality and correlations with range much smaller than $N$ could not be seen. On the other hand, choosing $N$ too small causes big fluctuations of the local value of the exponent [5].
There is no exact formula for calculating width value. For detailed analysis of impact of width on the results see [5] and recerences herein. The procedure continues as follows. Divide the first $N$ values of the integrated time series into windows of the same size $\tau$, which is chosen to be a divisor of $N$. Thus we will have $N / \tau$ windows. Apply linear fit in each window and then obtain linear approximation of the trend, denoted by $y(i)$, $i=1, \ldots, N$. Calculate the variance of the difference between the original integrated time series and the local trend in each window as

$$
F_{k}^{2}(\tau)=\frac{1}{\tau} \sum_{i=1}^{\tau}\left[x_{k}(i)-y_{k}(i)\right]^{2} .
$$

The above equation defines the variance in $k$-th window, $k=1, \ldots, N / \tau, x_{k}(i)-i$-th term in $k$-th window of the integrated time series. Average the variances over all windows

$$
\left\langle F^{2}(\tau)\right\rangle=\frac{\tau}{N} \sum_{k=1}^{\mathrm{N} / \tau} F_{k}^{2}(\tau) .
$$

Then, change the size of the window and repeat the above procedure. Obviously, the average variance will increase with window size. However, a power law behavior is expected [2]:

$$
\left\langle F^{2}(\tau)\right\rangle \propto \tau^{2 H_{\mathrm{loc}}}
$$

The above procedure allows one to calculate the local (210 prices) Hurst exponent on the session by session basis by moving the observation box. The extracted $H_{\text {loc }}$ measures the persistency of the time series $\left(H_{\text {loc }}<1 / 2\right)$ or antipersistency $\left(H_{\text {loc }}>1 / 2\right)$. Special case $H_{\text {loc }}=1 / 2$ corresponds to the Brownian motion [6].

\section{Data}

We analyze the daily index values of selected stock markets. In order to be able to perform preliminary comparison between emerging and mature markets we choose data from both of these groups. Polish and Hungary stock exchanges are representatives of emerging markets. 
On the other hand, we use data of NYSE and London Stock Exchange - very big and old financial markets, as well as French, Netherlander and German - mature but not so big markets. The same group contains also two other South American stock markets - Argentinean and Brazilian. Information on the analyzed data is collected in Table.

TABLE

Description of data.

\begin{tabular}{l|l|c|c}
\hline \hline Index & Stock Exchange & Start date & End date \\
\hline WIG & Poland (Warsaw) & $1991-04-16$ & $2009-04-30$ \\
DJIA & USA (New York) & $1995-01-03$ & $2009-04-30$ \\
DAX & Germany (Frankfurt) & $1995-01-02$ & $2009-04-30$ \\
BUX & Hungary (Budapest) & $1995-01-03$ & $2009-04-30$ \\
BOVESPA & Brazil (Sao Paulo) & $1995-01-02$ & $2009-09-14$ \\
BUENOS & Argentina (Buenos Aires) & $1996-10-08$ & $2009-09-14$ \\
CAC40 & France (Paris) & $1995-01-03$ & $2009-09-14$ \\
EOE & Netherlands (Amsterdam) & $1995-01-02$ & $2009-09-14$ \\
FT-SE100 & England (London) & $1992-10-22$ & $2009-09-14$
\end{tabular}

To give the idea of the differences between investigated markets, i.e., emerging vs. mature as well as small vs. big, we present stock exchanges in Fig. 1. The horizontal axis shows the year a stock exchange has been founded.

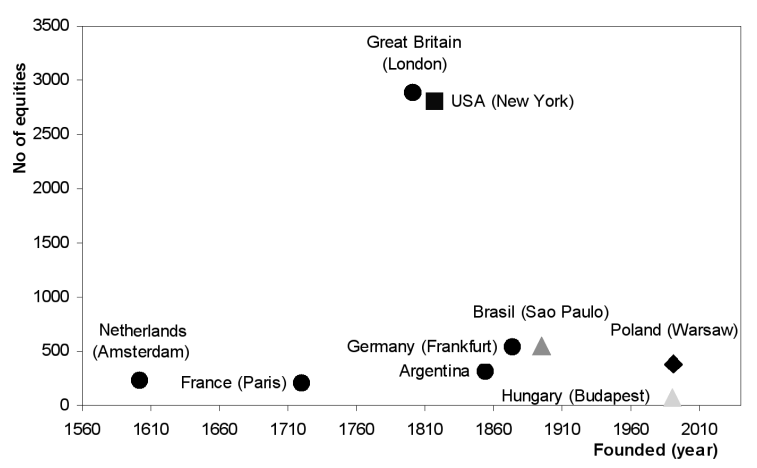

Fig. 1. Market development diagram.

In the case of Warsaw and Budapest stock exchanges we assume the year of foundation to be 1991 and 1990, respectively. Although Warsaw stock exchange existed before Second World War, there was a 40-year-break of operation during post war times. That is why we assume the above starting year of the "new" stock market. To display size of the market we decided to use number of equity noted (vertical axis). This choice could be questionable but a number of companies, annual turnover and daily volume usually come together. Choosing the number of companies as the indication of size allows us to avoid problems with comparison of different currencies, etc.

\section{Analysis}

There are works using local Hurst exponent to predict crashes on the financial markets (eg. [4, 7]). It has been noticed that decrease of $H_{\text {loc }}$ as well as other $H_{\text {loc }}$-related signals precede crash. These observations have been confirmed by the WIG (Poland) and DJIA (NYSE) signals as well as by analysis of some other markets [7]. As an example and as an introduction to further analysis we present (see Fig. 2) log-prices and local Hurst exponent for EOE index (Netherlands, Amsterdam). For convenience running average of 20 values of $H_{\text {loc }}$ has been plotted instead of values. There is the common horizontal scale for both plots. Crashes indicated by A, B, and $\mathrm{C}$ are clearly preceded by decrease of $H_{\text {loc }}$ to the values below 0.5 . Similar situation can be observed in the case of $\mathrm{D}$ and $\mathrm{E}$, but the Hurst exponent values decrease almost at the same time when index drops.

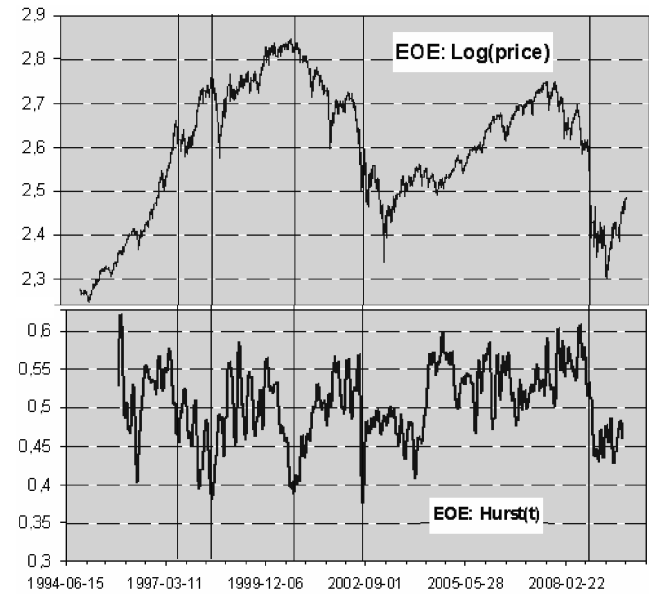

Fig. 2. EOE index $\log$ (price) vs. date (top) and local Hurst exponent (bottom). Vertical lines indicate crashes, see text.

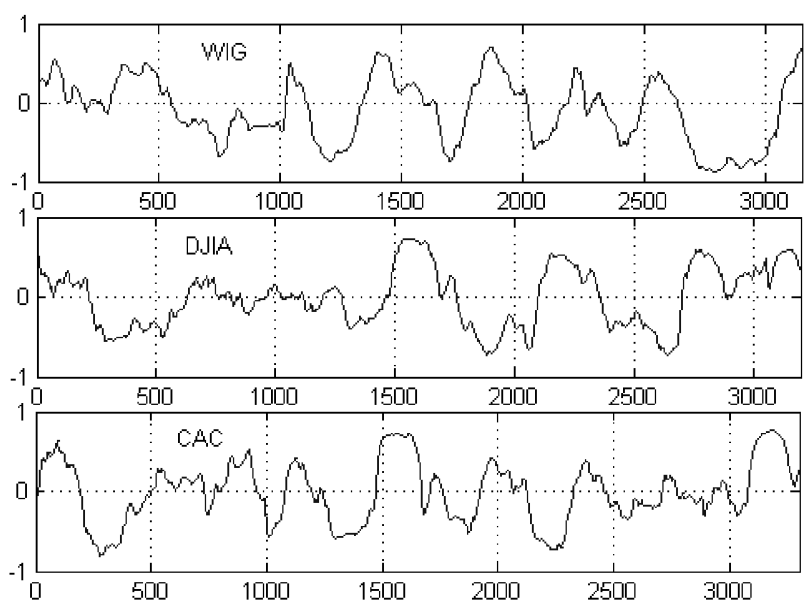

Fig. 3. Correlation coefficient between $\log ($ price $)$ and $H_{\text {loc }}$ for three markets. Vertical axis: arbitrary trading day number.

In order to compare exponent with prices in more quantitative way we calculated $C(i)$, i.e., correlation co- 
efficient between $H_{\text {loc }}$ and $\log ($ price $)$, given by the equation below

$$
C(i)=\frac{\operatorname{cov}\left(H_{\mathrm{loc}}, \log (\text { price })\right)}{\sigma_{H_{\mathrm{loc}}} \cdot \sigma_{\log (\text { price })}} .
$$

The running correlation coefficient $C(i)$ was calculated between $H_{\mathrm{loc}}(i-N+1) \div H_{\mathrm{loc}}(i)$ and $p(i-N+1) \div p(i)$, where $p(i)=\log (\operatorname{price}(i))$. The value of the index $(i)$ is chosen in such a way the $p(i)$ is the logarithmic price for the same day as $H_{\mathrm{loc}}$ calculated for $[i-N+1 ; i]$ in- tegrated price series. Correlation coefficients for WIG, DJIA, and $\mathrm{CAC}$ are presented in Fig. 3. The $C(i)$ varies from about -0.8 to 0.8 for all three markets, changing sign on the regular basis. Thus the Hurst exponent exhibits strong correlation with $\log ($ em price), frequently going into strong anti-correlation and vice versa. We decided to measure frequency of changes or in other words, time between switches.

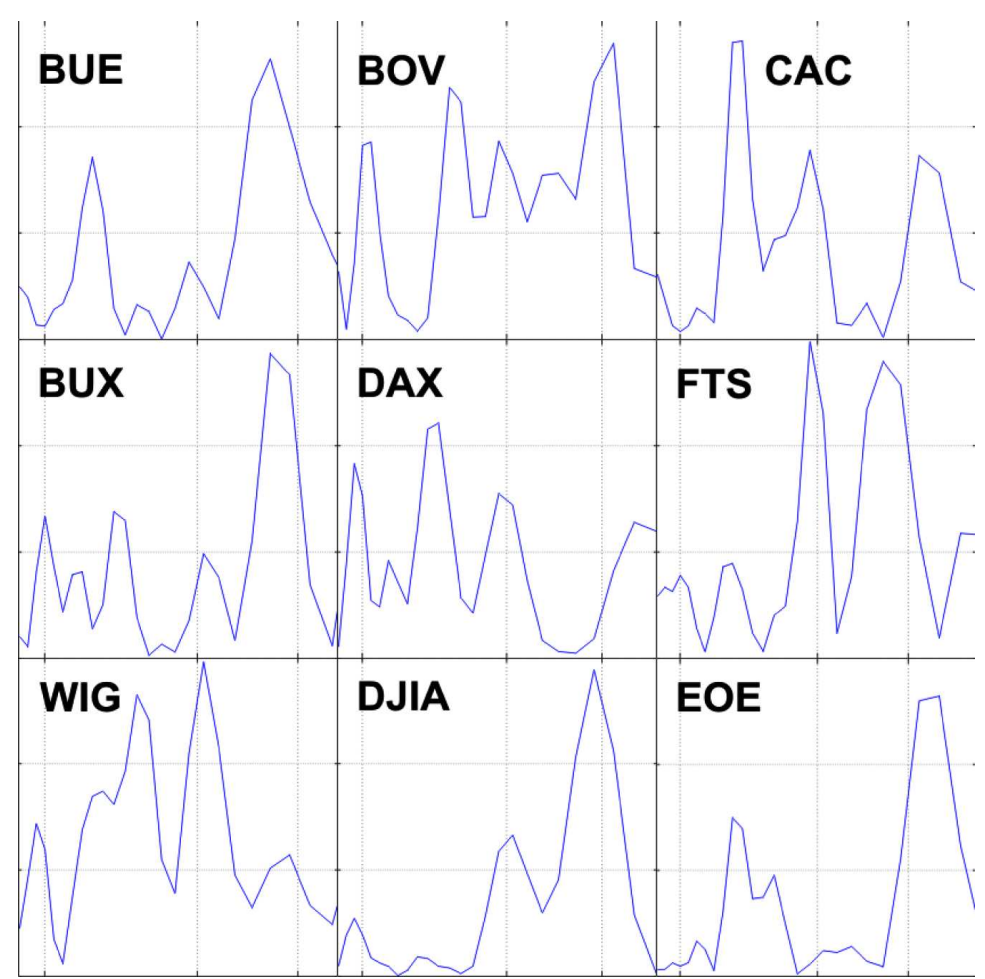

Fig. 4. Spectra of periods obtained using the discrete Fourier transform of the correlation function. Vertical dashed lines in each plot represent 300,500, 700 days period. Horizontal dashed lines are drawn in $1 / 3$ and $2 / 3$ of the total height of each plot. Vertical axes are in arbitrary units.

In order to quantitatively measure the periodicity of analyzed time series we use discrete Fourier transform. The common use of discrete Fourier transform (DFT) is to find the frequency components of a signal buried in a noisy time domain signal

$$
X(k)=\sum_{j=1}^{\mathrm{N}} x(j) \omega_{\mathrm{N}}^{(j-1)(k-1)},
$$

where $\omega_{\mathrm{N}}=\mathrm{e}^{(-2 \pi \mathrm{i}) / N}$. In order to present our results in more intuitive way we show magnitude of the Fourier transform as a function of period $T$, instead of frequency $k$ (see Fig. 4). Because the data consist of about 3000 prices, we studied periodic components with periods up to about 1000 days, so at least 3 periods could fit in the analyzed data.

In the case of some markets one can observe one or two big spikes indicating dominant periods. The one of the spikes is located (depending on the market) around 650-750 days (BUE, BUX, DJIA, EOE, FTS). For BOV that spike is visible, too. However, its height is obscured by the large background. In the case of CAC the spike is visible but not dominant, making this market similar to market represented by DAX index. These two indices (CAC - France, DAX - Germany) are similar by the presence of dominant spike around 350-400 days accompanied by another smaller one around 480-500 days. For the Polish market there are two spikes of different ratio but with similar positions.

The last part of this paper concerns characteristics of the spectra of local Hurst exponent. The mean value and standard deviation form the axes of the diagram in Fig. 5. As it can be seen from this picture, the central part is occupied by the group of big and long-operating markets: New York, Sao Paulo, Frankfurt, London, Am- 
sterdam. On the other hand there are also markets in the top-right part of the graph - big value of mean and standard deviation. Buenos Aires could be regarded as the market interpolating between East European emerging markets and well developed markets mentioned at the beginning of this paragraph. Indeed, Argentinean stock exchange has less importance in comparison with Brazilian one. The Paris stock exchange is located on the part of the graph characterized by relatively small mean and standard deviation of the $H_{\text {loc }}$.

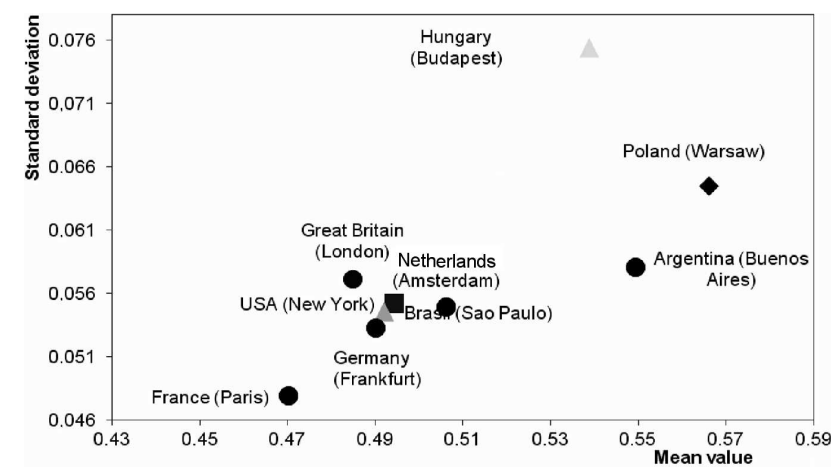

Fig. 5. Parameters of $H_{\text {loc }}$ distribution for analyzed markets.

\section{Summary}

Comparison of the $\log ($ price $)$ with local Hurst exponent for selected markets has been performed using correlation coefficient $C(i)$. Local (210 sessions) correlation coefficient exhibits periodicity. The periodic components of $C(i)$ have been evaluated using the discrete Fourier transform. Various similarities and differences between investigated stock markets have been pointed out. Characteristics of $H_{\text {loc }}$ spectra for analyzed markets have been briefly discussed.

\section{References}

[1] R. Weron, Physica A 332, 285 (2002).

[2] Y. Liu, P. Gopikrishnan, P. Cizeau, M. Meyer, C.K. Peng, H.E. Stanley, Phys. Rev. E 60, 1390 (1999).

[3] D. Grech, G. Pamuła, Physica A 387, 4299 (2008).

[4] D. Grech, Z. Mazur, Physica A 336, 133 (2004).

[5] C.K. Peng, S.V. Buldyrev, A.L. Goldberger, S. Havlin, M. Simons, H.E. Stanley, Phys. Rev. E 47, 3730 (1993).

[6] B.B. Mandelbrot, The Fractal Geometry of Nature, W.H. Freeman, New York 1982.

[7] P. Oświęcimka, J. Kwapien, S. Drożdż, R. Rak, Acta Phys. Pol. B 36, 2447 (2005). 